\title{
Actividad Antibacteriana y Citotoxicidad de Cinco Especies Vegetales de la Zona Altoandina y Amazónica de la Región del Cusco
}

\section{Antibacterial Activity and Cytotoxicity of Five Plant Species from the High Andean and Amazonian Zones of the Cusco Region}

Vera-Ferchau Karina ${ }^{1}$, Villena-Tejada Magaly ${ }^{1}$, Vera-Ferchau Ingrid $^{1} \&$ Cardona-Rivero Anahí ${ }^{1}$

\footnotetext{
${ }^{1}$ Escuela Profesional de Farmacia y Bioquímica. Facultad de Ciencias de la Salud. Universidad Nacional de San Antonio Abad del Cusco (UNSAAC).

*Correo electronico: karina.vera@unsaac.edu.pe.,magaly.villena@unsaac.edu.pe., ingrid. vera@unsaac.edu.pe., anahi.cardona@unsaac.edu.pe.
}

\section{Resumen}

La presente investigación, tuvo como objetivo evaluar la actividad antibacteriana y citotoxicidad de las especies Acicarpha tribuloides Jussieu (Estrella Kisca), Gamochaeta spicata (Queto-Queto), Minthostachys glabrescens (Bentham) Epling (Muña), Muehlenbeckia volcanica (Bentham) Endlicher (Mullaca) y Piper elongatum M. Vahl (Matico) de la zona altoandina y amazónica de la región del Cusco, Perú. Se desarrolló una investigación descriptiva, no experimental y transversal. Se prepararon extractos etanólicos mediante el método de maceración. La actividad antibacteriana, se determinó por medio de la concentración mínima inhibitoria (CMI), empleándose el método de macro dilución en medio líquido y cepas ATCC de Escherichia coli 25922, Staphylococcus aureus 29213, Streptococcus pneumoniae 49619 y Salmonella typhimurium 14028. Para determinar el grado de toxicidad se utilizó el ensayo de Artemia salina. Los resultados de la actividad antibacteriana mostraron que el Queto-Queto y la Mullaca tienen efecto frente a S.aureus, E. coli y S.pneumoniae, Mientras que, el Matico actúa frente a $S$.pneumoniae y $S$.aureus. En el caso de la Estrella Kisca y Muña dieron positivo frente a S.typhimurium y E.coli. La prueba de citotoxicidad con Artemia salina, demostró que, los extractos de Estrella Kisca y Queto Queto son muy tóxicos con una $\mathrm{CL}_{50}$ de 19.639 y 58.104 respectivamente. Por otro lado, los extractos considerados moderadamente tóxicos son los de Muña, Mullaca y Matico con $\mathrm{CL}_{50}$ de $100.092,415.451$ y 158.006 respectivamente. Se llegó a la conclusión que todos los extractos etanólicos tienen actividad antibacteriana y presentan diferente grado de toxicidad. 
Palabras clave: Acicarpha tribuloides, Gamochaeta spicata, Minthostachys glabrescens, Muehlenbeckia volcanica, Piper elongatum, Artemia salina, citotoxicidad, actividad antibacteriana.

\begin{abstract}
The objective of this research was to evaluate the antibacterial activity and cytotoxicity of the species Acicarpha tribuloides Jussieu (Estrella Kisca), Gamochaeta spicata (Queto-Queto), Minthostachys glabrescens (Bentham) Epling (Muña), Muehlenbeckia volcanica (Bentham) Endlicher (Mullaca) and Piper elongatum M. Vahl (Matico) from the high Andean and Amazonian zones of the Cusco region, Peru. A descriptive, non-experimental and cross-sectional research was carried out. Ethanolic extracts were prepared by the maceration method. The antibacterial activity was determined by means of the minimum inhibitory concentration (MIC), using the macro dilution method in liquid medium and ATCC strains of Escherichia coli 25922, Staphylococcus aureus 29213, Streptococcus pneumoniae 49619 and Salmonella typhimurium 14028. The Artemia salina assay was used to determine the degree of toxicity. The results of the antibacterial activity showed that Queto-Queto and Mullaca have effect against S.aureus, E. coli and S.pneumoniae, while, Matico acts against S.pneumoniae and S.aureus. In the case of Estrella Kisca and Muña, they were positive against S.typhimurium and E.coli. The cytotoxicity test with Artemia salina showed that the extracts of Estrella Kisca and Queto - Queto are very toxic with an $\mathrm{LC}_{50}$ of 19,639 and 58,104 respectively. On the other hand, the extracts considered moderately toxic are those of Muña, Mullaca and Matico with $\mathrm{LC}_{50}$ of 100,$092 ; 415,451$ and 158,006 respectively. It was concluded that all ethanolic extracts have antibacterial activity and have different degree of toxicity.
\end{abstract}

Key words: Acicarpha tribuloides, Gamochaeta spicata, Minthostachys glabrescens, Muehlenbeckia volcanica, Piper elongatum, Artemia salina, cytotoxicity, antibacterial activity.

\title{
Introducción
}

En los últimos años el uso de plantas medicinales se ha visto incrementado en gran medida (Aguirre et al., 2016). Es así, que en la región Cusco existe una alta riqueza de plantas medicinales comercializadas en los mercados y cuyos conocimientos ancestrales están arraigados en la cultura quechua, de las cuales $83 \%$ son especies nativas y un $78 \%$ proceden de localidades aledañas a la región andina (Huamantupa et al.,2011).

La flora representa un $10 \%$ del total mundial, del cual un $30 \%$ es endémico. El Perú es el quinto país en el mundo en número de plantas conocidas y usadas por la 
población; y es el primero en especies domesticadas nativas (182 especies) (Brack, 1999). Bajo esa premisa, el estado peruano a través de EsSalud, promociona el uso de las plantas medicinales como alternativa o medicina complementaria.

Según la Organización Mundial de la Salud (2002), para el futuro, la información fidedigna en cuanto al uso apropiado de productos y terapias será el reto más importante para la Medicina Tradicional/Medicina Alternativa- Complementaria. La transculturación había resultado en una pérdida grande del conocimiento tradicional de plantas silvestres de gran valor para la ciencia y la tecnología del país (Brack, 1999). En vista, que la identificación y los valores nacionales son reafirmados por el conocimiento vegetal de todos los grupos étnicos de Perú, es de gran importancia que no se pierdan por la modernización y globalización (Bussmann, R. W., \& Sharon, D., 2016).

En ese sentido, el desarrollo de la moderna investigación farmacológica va dirigido al estudio de las propiedades de plantas medicinales; precisamente la perspectiva más inmediata que se entrelaza dentro de una visión integral que facilite el desarrollo de una agenda para el adecuado uso de plantas medicinales es la investigación de sus propiedades y posteriormente las posibilidades de desarrollo de fitofármacos (Salaverry, 2005).

En nuestra región son escasos los reportes de investigaciones sobre medicina tradicional y etnobotánica, razón por la cual el objetivo de esta investigación fue evaluar la actividad antibacteriana y citotoxicidad de cinco especies vegetales de la zona altoandina y amazónica de la región del Cusco.

\section{Material y métodos}

\section{Recolección y Preparación de las Muestras Vegetales}

Se trabajó con las hojas de cinco especies vegetales de la región del Cusco como son Acicarpha tribuloides Jussieu (Estrella Kisca) recolectada en provincia el distrito de Santiago, localidad Occopata-Ancaschaca, altitud $3922 \mathrm{msnm}$ y coordenadas UTM19L-175987-8494223, Gamochaeta spicata (Queto-Queto) recolectada en la provincia del Cusco, distrito Santiago, localidad Occopata-Ancaschaca altitud $3950 \mathrm{msnm}$, coordenadas UTM-19L-175987-8494236; Minthostachys glabrescens (Bentham) Epling (Muña) recolectada en la provincia de Calca, distrito Yucay, localidad alturas de Yucay con coordenadas UTM-19L-189848-8513312; Muehlenbeckia volcanica (Bentham) Endlicher (Mullaca) recolectada en la provincia de Paruro, distrito Huaroquite, localidad Huanca-Huanca, coordenadas UTM-18L-814748-847859 y Piper elongatum M. Vahl (Matico) se recolectó en la provincia de La Convención, distrito Santa Ana, localidad Santa Teresa, altitud $800 \mathrm{msnm}$, coordenadas UTM-18L-758430-8586152. Los ejemplares fueron identificados en el Herbario Vargas Cuz de la Universidad Nacional de San Antonio Abad del Cusco. Las muestras se secaron en un secadero de plantas al medio ambiente y en sombra durante 15 días. El material seco se homogenizó utilizando un molino de martillos marca Si-lab modelo MM1.5 con tamaño de partícula $0.5 \mathrm{~mm}$ (USP38-NF33, 2015). 


\section{Preparación de los Extractos Hidroalcohólicos al 70\%}

De las muestras obtenidas de la molienda se tomaron 50 gramos de cada una y se colocaron en frascos de vidrio de boca ancha y de color ámbar, luego se agregó 500 $\mathrm{mL}$ de etanol de $70^{\circ} \mathrm{y}$ fueron llevados a maceración a temperatura ambiente por 15 días, agitando manualmente durante 5 minutos dos veces al día. Luego, se filtró el macerado utilizando papel Whatman $\mathrm{N}^{\circ} 1$ en un matraz estéril, el filtrado fue llevado al evaporador de extractos marca Si-lab, modelo EE $80 \mathrm{~L}$ a $40^{\circ} \mathrm{C}$ hasta la evaporación total, obteniéndose el extracto seco de cada planta (USP38-NF33, 2015).

\section{Preparación de los Aceites Esenciales}

La extracción del aceite esencial de Minthostachys glabrescens (Bentham) Epling (Muña), se realizó en un destilador por arrastre de vapor de agua marca Si-lab, modelo DAE20L, para lo cual se utilizó $5 \mathrm{~kg}$. de la planta seca.

\section{Procedimiento para el Análisis Fitoquímico Cualitativo}

Para la identificación cualitativa de los metabolitos secundarios se utilizó el tamizaje fitoquímico de Rondina y Coussio (1969), modificado por Alfonso et al. (2000). A una porción de un gramo del extracto seco de cada especie vegetal, se le realizaron reacciones de coloración y/o precipitación con diferentes agentes cromóforos específicos para los siguientes grupos funcionales: flavonoides, compuestos fenólicos, quinonas, resinas, alcaloides, taninos y saponinas. En el análisis cualitativo se utilizó el sistema de cruces ( + poca cantidad, ++ cantidad moderada y +++ abundante cantidad) y se detalló la presencia o ausencia de los metabolitos secundarios en las muestras. (USP38-NF33, 2015).

\section{Procedimiento para el Análisis Espectrofotométrico UV - Visible}

Para el análisis espectrofotométrico, se utilizó un espectrofotómetro UV-Vis doble haz, marca Jenway, modelo 6850 . Se preparó una infusión al $10 \%$ con agua destilada y posteriormente, se tomó una muestra de $3 \mathrm{~mL}$ de la infusión para realizar el análisis en el espectrofotómetro UV VIS en el rango de 190-800 nm. (USP38-NF33, 2015).

\section{Determinación del Efecto Antibacteriano}

Se determinó la concentración mínima inhibitoria (CMI) antibacteriana para lo cual se utilizó el método de macro dilución en medio líquido (Granados R. y Villaverde C., 1997; Woods G y Washington J. 1995).Para lo cual se utilizaron cepas de ATCC de Escherichia coli 25922, Staphylococcus aureus 29213, Streptococcus pneumoniae 49619 y Salmonella typhimurium 14028, las que fueron conservadas en tubos con $40 \mathrm{~mL}$ de agar tripticasa soya en pico de flauta debidamente rotulados, codificados e incubados a $37^{\circ} \mathrm{C}$ por 24 horas, para luego conservar a $4^{\circ} \mathrm{C}$ y repicar a un nuevo medio cada 15 días para la conservación de la cepa. Luego se procedió a la activación de la cepa, seguidamente se realizó la estandarización de las curvas de crecimiento de las cepas, para lo cual se tomó el inóculo de la muestra de la cepa, se propagó en BHIA (Brain and Heard Infusión Agar) e incubó a $37^{\circ} \mathrm{C}$ por 24 horas. Para estandarizar la concentración de la cepa se comparó con una escala de Mac Farland de 0.5 que equivale a $1.5 \times 10^{8} \mathrm{UFC} / \mathrm{mL}$. 
En el caso de estrella kiska, queto-queto, mullaca, matico se trabajó con los extractos secos etanólicos, para lo cual, se realizaron una serie de diluciones con caldo Muller Hinton en 10 concentraciones de 5.12, 2.56, 1.28, 0.64, 0.32, 0.16, 0.08, 0.04, 0.02 y $0.01 \mathrm{mg} / \mathrm{mL}$.

En lo referente a la muña se trabajó con el aceite esencial en concentraciones porcentuales de 50, 25, 12.5, 6.25, 3.13, 1.56, 0.78, 0.39, 0.19, 0.095\%. En tubos con tapón rosca, se colocaron un mililitro de cada solución de extractos y un mililitro de caldo de cultivo estéril. Luego se inoculó con un mililitro de suspensión de las cuatro cepas bacterianas en cada tubo, se incubó por 24 horas a $37^{\circ} \mathrm{C}$. Luego se determinó la concentración mínima inhibitoria (CMI).

\section{Determinación de la Citotoxicidad con Artemia salina}

El ensayo de Artemia salina ha sido utilizado para determinar la presencia de componentes citotóxicos en los extractos vegetales. Los nauplios del crustáceo Artemia salina son muy sensibles a una gran variedad de sustancias químicas, lo cual sirve para determinar el grado de toxicidad de los extractos según el valor de la Concentración Letal Media $\left(\mathrm{CL}_{50}\right)$.

Para determinar el grado de toxicidad de los extractos usando el ensayo de Artemia salina, se realizó en función del rango en que se encontraron los valores de $\mathrm{CL}_{50}$ de acuerdo con las categorías siguientes: extremadamente tóxico $\left(\mathrm{CL}_{50}<10 \mu \mathrm{g} / \mathrm{mL}\right)$, muy tóxico $\left(10<\mathrm{CL}_{50}<100\right)$, moderadamente tóxico $\left(100<\mathrm{CL}_{50}<1000 \mu \mathrm{g} / \mathrm{mL}\right)$ y no tóxico $\left(\mathrm{CL}_{50}>1000 \mu \mathrm{g} / \mathrm{mL}\right)$. (CYTED, 1995).

\section{Preparación de Extractos y Adición de Larvas}

Este ensayo se realiza en varias etapas: (CYTED, 1995)

El primer día se preparan los extractos, disolviendo $0,025 \mathrm{~g}$ del extracto seco en $25 \mathrm{~mL}$ de agua de mar artificial, la cual se prepara con $3.8 \mathrm{~g}$ de sal sin yodo (sal de Maras), en $100 \mathrm{~mL}$ de agua destilada, luego se filtra. Se coloca $50 \mathrm{mg}$ de huevos de Artemia salina Leach en el agua de mar artificial con luz permanente y a una temperatura promedio de $28^{\circ} \mathrm{C}$ por un periodo de 24 horas para la eclosión de los huevos. Para cada muestra se usan 12 tubos de ensayo distribuidos de la siguiente manera: 3 tubos de ensayo para el grupo blanco, 3 tubos ensayo para la concentración de 10 ppm, 3 para 100 ppm y 3 para $1000 \mathrm{ppm}$.

En el segundo día, se pesan $0.025 \mathrm{~g}$ del extracto seco y se procede a realizar las diluciones, así para la dilución de 1000 ppm, se disuelven $0.025 \mathrm{~g}$ del extracto seco en $25 \mathrm{~mL}$ de agua de mar artificial (solución A), la cual se divide en 3 tubos de ensayo cada uno de $4 \mathrm{~mL}$. Para la obtención de la dilución de $100 \mathrm{ppm}$, se toman $10 \mathrm{~mL}$ de la solución A y se afora a $100 \mathrm{~mL}$ (solución B). Luego se divide en 3 tubos de ensayo (4 mL para cada tubo de ensayo). Para la obtención de 10 ppm de la solución $\mathrm{B}$ se toman $10 \mathrm{~mL}$ aforándose a $100 \mathrm{~mL}$ y se divide en 3 tubos de ensayo ( $4 \mathrm{~mL}$ para cada tubo de ensayo). 
Obtenidas las diluciones, se procede a la transferencia de 10 nauplios de Artemia salina Leach a los tubos de ensayo, utilizando pipetas Pasteur.

Los tubos se colocan en cámaras de poca iluminación y temperatura adecuada, durante 24 horas. Luego de este tiempo se realiza el conteo de sobrevivientes y muertos. Se utilizan grupos blanco, donde los tubos sólo contienen nauplios en agua de mar artificial, en las mismas condiciones que las anteriores. A todos los tubos (incluyendo los controles) se adiciona una gota de suspensión de levadura comercial como fuente de alimento (solución de $0.06 \%$ levadura seca por litro). Cada tubo alcanza un volumen final de $5 \mathrm{~mL}$.

En el tercer día, se realiza el recuento del número de nauplios sobrevivientes en cada dilución. Para determinar la $\mathrm{CL}_{50}$ con $95 \%$ de intervalo de confianza, se realizan los cálculos utilizando el método de análisis de Probit.

\section{Resultados}

Tabla 1

Resultados de las pruebas fitoquímicas cualitativas de las especies en estudio

\begin{tabular}{|c|c|c|c|c|c|}
\hline Tipo de prueba & $\begin{array}{l}\text { Acicarpha } \\
\text { tribuloides } \\
\text { Jussieu } \\
\text { (Estrella } \\
\text { Kisca) }\end{array}$ & $\begin{array}{l}\text { Gamochaeta } \\
\text { spicata } \\
\text { (Queto-Queto) }\end{array}$ & $\begin{array}{l}\text { Minthostachys } \\
\text { glabrescens } \\
\text { (Bentham) } \\
\text { Epling (Muña) }\end{array}$ & $\begin{array}{l}\text { Muehlenbeckia } \\
\text { volcanica } \\
\text { (Bentham) } \\
\text { Endlicher } \\
\text { (Mullaca) }\end{array}$ & $\begin{array}{l}\text { Piper } \\
\text { elongatum } \\
\text { M. Vahl } \\
\text { (Matico) }\end{array}$ \\
\hline Flavonoides & positivo & positivo & positivo & positivo & positivo \\
\hline $\begin{array}{l}\text { Compuestos } \\
\text { fenólicos }\end{array}$ & positivo & positivo & positivo & positivo & positivo \\
\hline Quinonas & negativo & negativo & negativo & negativo & negativo \\
\hline Resinas & positivo & positivo & negativo & negativo & positivo \\
\hline Alcaloides & positivo & positivo & positivo & positivo & positivo \\
\hline Taninos & positivo & positivo & positivo & positivo & positivo \\
\hline Saponinas & positivo & positivo & positivo & positivo & positivo \\
\hline $\begin{array}{l}\text { Lactonas } \\
\text { sesquiterpénicas }\end{array}$ & negativo & negativo & negativo & positivo & negativo \\
\hline
\end{tabular}

En la Tabla 1, muestra el análisis fitoquímico cualitativo realizado en el extracto seco de las cinco especies vegetales en estudio, donde se observa que las cinco plantas investigadas presentan en su composición flavonoides, compuestos fenólicos, alcaloides, taninos y saponinas. Las plantas Acicarpha tribuloides Jussieu (Estrella Kisca), Gamochaeta spicata (Queto-Queto) y Piper elongatum M. Vahl (Matico) contienen 
resinas; mientras que, las lactonas sesquiterpénicas han dado positivo solamente para la especie Muehlenbeckia volcanica (Bentham) Endlicher (Mullaca). En ninguna de las plantas se han identificado las quinonas.

\section{Tabla 2}

Resultados del análisis espectrofotométrico $U V$ - visible del extracto seco de las especies vegetales investigadas

\begin{tabular}{|c|c|c|c|c|c|c|}
\hline $\begin{array}{l}\text { Longitud } \\
\text { de onda }\end{array}$ & $\begin{array}{c}\text { Acicarpha } \\
\text { tribuloides } \\
\text { Jussieu } \\
\text { (Estrella } \\
\text { Kisca) }\end{array}$ & $\begin{array}{c}\text { Piper } \\
\text { elongatum M. } \\
\text { Vahl (Matico) }\end{array}$ & $\begin{array}{c}\text { Muehlenbeckia } \\
\text { volcanica } \\
\text { (Bentham) } \\
\text { Endlicher } \\
\text { (Mullaca) }\end{array}$ & $\begin{array}{l}\text { Minthostachys } \\
\text { glabrescens } \\
\text { (Bentham) } \\
\text { Epling (Muña) }\end{array}$ & $\begin{array}{c}\text { Gamochaeta } \\
\text { spicata (Queto- } \\
\text { Queto) }\end{array}$ & $\begin{array}{l}\text { Metabolitos } \\
\text { secundarios }\end{array}$ \\
\hline$\lambda$ & Absorbancia & Absorbancia & Absorbancia & Absorbancia & Absorbancia & \\
\hline 200 & 0.9125 & & & 0.7549 & & flavonoides \\
\hline 210 & & 0.9987 & 0.9718 & 0.9489 & & saponinas \\
\hline 225 & & 1.0838 & 1.1331 & & & $\begin{array}{l}\text { Sesquiterpenlactonas } \\
\alpha, \beta \text { Insaturadas }\end{array}$ \\
\hline 232 & 1.1544 & & & & 1.0818 & flavonas \\
\hline 236 & 1.0416 & & & & 1.0697 & flavonoides \\
\hline 250 & 0.9584 & 0.9343 & & 0.8673 & & $\begin{array}{l}\text { terpenos compuestos } \\
\text { aromáticos, fenol }\end{array}$ \\
\hline 260 & 0.9728 & & & & & flavonoides \\
\hline 270 & 1.2455 & & & & 1.1570 & $\begin{array}{l}\text { terpenos compuestos } \\
\text { aromáticos, fenol }\end{array}$ \\
\hline 275 & & & 1.2308 & & & flavonoides \\
\hline 279 & 1.1431 & & & & & flavonas \\
\hline
\end{tabular}

Fuente: Elaboración propia basada en datos experimentales

En la tabla 2, se aprecian las longitudes de onda en las cuales se observaron picos de absorción para los diferentes metabolitos secundarios, donde las especies Estrella Kisca y Muña mostraron picos a $200 \mathrm{~nm}$ correspondiente a los flavonoides. Las saponinas que absorben a $210 \mathrm{~nm}$ están presentes en las especies Matico, Mullaca y Muña. A 225 nm en la cual absorben las lactonas sesquiterpénicas $\alpha, \beta$ insaturadas, mostraron picos de absorción el Matico y la Mullaca. Mientras que, a $232 \mathrm{~nm}$ y $236 \mathrm{~nm}$, longitudes de onda para flavonas y flavonoides, observamos picos para la Estrella Kisca y el Queto-Queto. En el caso de los compuestos fenólicos que absorben a longitudes de onda de $250 \mathrm{y}$ $270 \mathrm{~nm}$, fueron identificados los picos para las especies Estrella Kisca, Matico, Muña y Queto-Queto. Los flavonoides (275 nm) se encontraron en la Mullaca y las flavonas (279 nm) en la Estrella Kisca. 
Tabla 3

Evaluación de actividad antibacteriana a de extractos de especies vegetales y aceite esencial de Muña

\begin{tabular}{|c|c|c|c|c|c|c|c|c|}
\hline \multirow[b]{2}{*}{ Especie vegetal } & \multicolumn{2}{|c|}{$\begin{array}{l}\text { Staphylococcus } \\
\text { aureus }\end{array}$} & \multicolumn{2}{|c|}{ Escherichia coli } & \multicolumn{2}{|c|}{$\begin{array}{l}\text { Streptococcus } \\
\text { pneumoniae }\end{array}$} & \multicolumn{2}{|c|}{$\begin{array}{l}\text { Salmonella } \\
\text { typhimurium }\end{array}$} \\
\hline & Efecto & CMI & Efecto & CMI & Efecto & CMI & Efecto & CMI \\
\hline $\begin{array}{l}\text { Acicarpha } \\
\text { tribuloides } \\
\text { Jussieu (Estrella } \\
\text { Kisca) }\end{array}$ & Negativo & 0 & Positivo & $\begin{array}{l}2.56 \mathrm{mg} / \\
\mathrm{mL}\end{array}$ & Negativo & 0 & Positivo & $\begin{array}{l}2.56 \mathrm{mg} / \\
\mathrm{mL}\end{array}$ \\
\hline $\begin{array}{l}\text { Gamochaeta } \\
\text { spicata (Queto- } \\
\text { Queto) }\end{array}$ & Positivo & $\begin{array}{l}1.28 \mathrm{mg} / \\
\mathrm{mL}\end{array}$ & Positivo & $\begin{array}{l}2.56 \mathrm{mg} / \\
\mathrm{mL}\end{array}$ & Positivo & $\begin{array}{l}1.28 \mathrm{mg} / \\
\mathrm{mL}\end{array}$ & Negativo & 0 \\
\hline $\begin{array}{l}\text { Muehlenbeckia } \\
\text { volcanica } \\
\text { (Bentham) } \\
\text { Endlicher } \\
\text { (Mullaca) }\end{array}$ & Positivo & $\begin{array}{l}0.64 \mathrm{mg} / \\
\mathrm{mL}\end{array}$ & Positivo & $\begin{array}{l}2.56 \mathrm{mg} / \\
\mathrm{mL}\end{array}$ & Positivo & $\begin{array}{l}0.64 \mathrm{mg} / \\
\mathrm{mL}\end{array}$ & Negativo & 0 \\
\hline $\begin{array}{l}\text { Piper } \\
\text { elongatum M. } \\
\text { Vahl (Matico) }\end{array}$ & Positivo & $\begin{array}{l}0.64 \mathrm{mg} / \\
\mathrm{mL}\end{array}$ & Negativo & 0 & Positivo & $\begin{array}{l}1.28 \mathrm{mg} / \\
\mathrm{mL}\end{array}$ & Negativo & 0 \\
\hline $\begin{array}{l}\text { Minthostachys } \\
\text { glabrescens } \\
\text { (Bentham) } \\
\text { Epling (Muña)* }\end{array}$ & Negativo & $0.00 \%$ & Positivo & $0.78 \%$ & Positivo & $0.78 \%$ & Positivo & $0.39 \%$ \\
\hline
\end{tabular}

*Se utilizó para el estudio antibacteriano aceite esencial.

En la tabla 3, se pueden observar los resultados de efecto antibacteriano de cinco especies vegetales frente a cuatro cepas bacterianas ATCC dos gram positivas (Staphylococcus aureus 29213, Streptococcus pneumoniae 49619) y dos gram negativas (Escherichia coli 25922 y Salmonella typhimurium). Se trabajaron con los extractos etanólicos y en caso de muña con el aceite esencial. 
Acicarpha tribuloides Jussieu (Estrella Kisca) tiene efecto antibacteriano contra gram negativos para Salmonella typhimurium y Escherichia coli (CMI $2.56 \mathrm{mg} / \mathrm{mL}$ ) y ausencia de actividad antibacteriana frente a gram positivos.

Gamochaeta spicata (Queto-Queto), presenta actividad antibacteriana frente al gram negativo Escherichia coli (CMI $2.56 \mathrm{mg} / \mathrm{dL}$ ) no tiene efecto frente a Salmonella typhimurium. En cuanto a gram positivos tiene efecto antibacteriano frente a Staphylococcus aureus (CMI $1.28 \mathrm{mg} / \mathrm{mL}$ ) y Streptococos pneumoniae (CMI $1.28 \mathrm{mg}$ / $\mathrm{mL}$ ).

El aceite esencial de Minthostachys glabrescens (Bentham) Epling (Muña), tiene efecto antibacteriano frente a gram positivos y gram negativos a excepción de Staphylococcus aureus.

Muehlenbeckia volcanica (Bentham) Endlicher (Mullaca), tiene efecto antibacteriano frente a Streptococcus pneumoniae (CMI: $0.64 \mathrm{mg} / \mathrm{mL}$ ), Staphylococcus aureus (CMI: $0.64 \mathrm{mg} / \mathrm{mL}$ ) y Escherichia coli (CMI: $2.56 \mathrm{mg} / \mathrm{mL}$ ). No tiene efecto antibacteriano frente a Salmonella typhimurium.

Piper elongatum M. Vahl (Matico), no tiene actividad antibacteriana contra los gram negativos Escherichia coli y Salmonella typhimurium. Tiene actividad antibacteriana contra gram positivos Staphylococcus aureus (CMI $0.64 \mathrm{mg} / \mathrm{mL}$ ) y Streptococos pneumoniae $(1.28 \mathrm{mg} / \mathrm{mL})$. 
Tabla 4

Prueba de citotoxicidad con Artemia salina Leach con los extractos secos en estudio

\begin{tabular}{|c|c|c|c|c|c|}
\hline & Dilución & Ensayo & $\begin{array}{l}\text { Total de nauplios } \\
\text { antes del ensayo }\end{array}$ & $\begin{array}{l}\text { Total de nauplios } \\
\text { vivos después del } \\
\text { ensayo }\end{array}$ & $\begin{array}{l}\text { Porcentaje de } \\
\text { nauplios vivos }\end{array}$ \\
\hline$\pi$ & \multirow{3}{*}{1000 ppm } & 1 & 10 & 0 & \multirow{3}{*}{0.00} \\
\hline $\bar{\Xi}$ & & 2 & 10 & 0 & \\
\hline 迅 & & 3 & 10 & 0 & \\
\hline $\overrightarrow{0}$ & \multirow{3}{*}{100 ppm } & 1 & 10 & 5 & \multirow{3}{*}{40.00} \\
\hline$\stackrel{n}{\underline{0}}$ & & 2 & 10 & 4 & \\
\hline \multirow{7}{*}{ 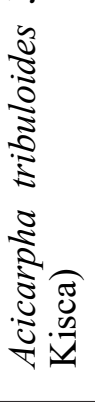 } & & 3 & 10 & 3 & \\
\hline & \multirow{3}{*}{10 ppm } & 1 & 10 & 5 & \\
\hline & & 2 & 10 & 4 & \multirow{2}{*}{43.33} \\
\hline & & 3 & 10 & 4 & \\
\hline & \multirow{3}{*}{ Control } & 1 & 10 & 9 & \multirow{3}{*}{96.67} \\
\hline & & 2 & 10 & 10 & \\
\hline & & 3 & 10 & 10 & \\
\hline \multirow{12}{*}{ 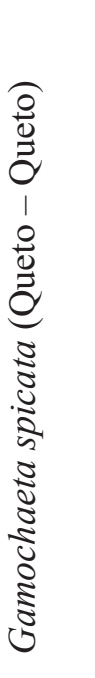 } & \multirow{3}{*}{$1000 \mathrm{ppm}$} & 1 & 10 & 0 & \multirow{3}{*}{0.00} \\
\hline & & 2 & 10 & 0 & \\
\hline & & 3 & 10 & 0 & \\
\hline & \multirow{3}{*}{100 ppm } & 1 & 10 & 3 & \multirow{3}{*}{16.67} \\
\hline & & 2 & 10 & 2 & \\
\hline & & 3 & 10 & 0 & \\
\hline & \multirow{3}{*}{$10 \mathrm{ppm}$} & 1 & 10 & 8 & \multirow{3}{*}{86.67} \\
\hline & & 2 & 10 & 9 & \\
\hline & & 3 & 10 & 9 & \\
\hline & \multirow{3}{*}{ Control } & 1 & 10 & 10 & \multirow{3}{*}{96.67} \\
\hline & & 2 & 10 & 10 & \\
\hline & & 3 & 10 & 9 & \\
\hline
\end{tabular}




\begin{tabular}{|c|c|c|c|c|c|}
\hline & Dilución & Ensayo & $\begin{array}{l}\text { Total de nauplios } \\
\text { antes del ensayo }\end{array}$ & $\begin{array}{l}\text { Total de nauplios } \\
\text { vivos después del } \\
\text { ensayo }\end{array}$ & $\begin{array}{l}\text { Porcentaje de } \\
\text { nauplios vivos }\end{array}$ \\
\hline \multirow{4}{*}{ 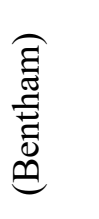 } & \multirow{3}{*}{1000 ppm } & 1 & 10 & 3 & \multirow{3}{*}{16.67} \\
\hline & & 2 & 10 & 2 & \\
\hline & & 3 & 10 & 0 & \\
\hline & \multirow{3}{*}{100 ppm } & 1 & 10 & 3 & \multirow{3}{*}{43.33} \\
\hline \multirow{3}{*}{ 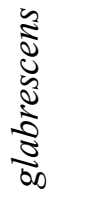 } & & 2 & 10 & 4 & \\
\hline & & 3 & 10 & 6 & \\
\hline & \multirow{3}{*}{10 ppm } & 1 & 10 & 5 & \multirow{3}{*}{46.67} \\
\hline \multirow{5}{*}{  } & & 2 & 10 & 6 & \\
\hline & & 3 & 10 & 7 & \\
\hline & \multirow{3}{*}{ Control } & 1 & 10 & 8 & \multirow{3}{*}{86.67} \\
\hline & & 2 & 10 & 8 & \\
\hline & & 3 & 10 & 10 & \\
\hline \multirow{4}{*}{ 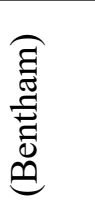 } & \multirow{3}{*}{1000 ppm } & 1 & 10 & 0 & \multirow{3}{*}{10.00} \\
\hline & & 2 & 10 & 0 & \\
\hline & & 3 & 10 & 3 & \\
\hline & \multirow{3}{*}{100 ppm } & 1 & 10 & 8 & \multirow{3}{*}{80.00} \\
\hline \multirow{8}{*}{ 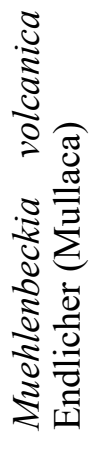 } & & 2 & 10 & 8 & \\
\hline & & 3 & 10 & 8 & \\
\hline & \multirow{3}{*}{10 ppm } & 1 & 10 & 8 & \multirow{3}{*}{76.67} \\
\hline & & 2 & 10 & 6 & \\
\hline & & 3 & 10 & 9 & \\
\hline & \multirow{3}{*}{ Control } & 1 & 10 & 9 & \multirow{3}{*}{86.67} \\
\hline & & 2 & 10 & 7 & \\
\hline & & 3 & 10 & 10 & \\
\hline \multirow{6}{*}{ 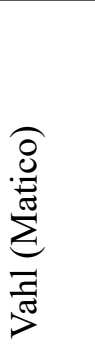 } & \multirow{3}{*}{1000 ppm } & 1 & 10 & 1 & \multirow{3}{*}{13.33} \\
\hline & & 2 & 10 & 2 & \\
\hline & & 3 & 10 & 1 & \\
\hline & \multirow{3}{*}{100 ppm } & 1 & 10 & 3 & \multirow{3}{*}{40.00} \\
\hline & & 2 & 10 & 5 & \\
\hline & & 3 & 10 & 4 & \\
\hline \multirow{6}{*}{ 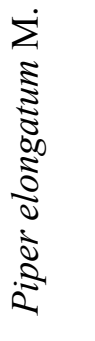 } & \multirow{3}{*}{10 ppm } & 1 & 10 & 5 & \\
\hline & & 2 & 10 & 8 & 70.00 \\
\hline & & 3 & 10 & 8 & \\
\hline & & 1 & 10 & 9 & \\
\hline & Control & 2 & 10 & 9 & 90.00 \\
\hline & & 3 & 10 & 9 & \\
\hline
\end{tabular}

Fuente: Elaboración propia basada en datos experimentales 
En la tabla 4, se muestra los resultados de la prueba de citotoxicidad con Artemia salina Leach de los extractos, donde se puede evidenciar claramente que todos los extractos de las especies estudiadas muestran la mayor mortalidad a las 24 horas a la concentración de 1000 ppm, ya que a esta concentración el porcentaje de nauplios sobrevivientes fue el menor para todos los casos.

\section{Tabla 5}

Porcentaje de mortalidad y Concentración letal media de los extractos secos en estudio

\begin{tabular}{|c|c|c|c|c|}
\hline & \multicolumn{3}{|c|}{ Porcentaje promedio de mortalidad a las } & \multirow{4}{*}{$\begin{array}{l}\text { Concentracion letal media } \\
u g / m L\end{array}$} \\
\hline & \multirow{3}{*}{$\begin{array}{l}24 \text { horas } \\
10 \mathrm{ppm} \\
0.01 \mathrm{mg} / \mathrm{mL}\end{array}$} & \multirow{3}{*}{$\begin{array}{l}100 \mathrm{ppm} \\
0.1 \mathrm{mg} / \mathrm{mL}\end{array}$} & \multirow{3}{*}{$\begin{array}{l}1000 \mathrm{ppm} \\
1 \mathrm{mg} / \mathrm{mL}\end{array}$} & \\
\hline & & & & \\
\hline & & & & \\
\hline Acicarpha tribuloides & & & & \\
\hline Jussieu (Estrella Kisca) & 55.17 & 58.62 & 100 & 19.639 \\
\hline $\begin{array}{l}\text { Gamochaeta spicata (Queto } \\
\text { - Queto) }\end{array}$ & 10.34 & 82.75 & 100 & 58.104 \\
\hline $\begin{array}{l}\text { Minthostachys glabrescens } \\
\text { (Bentham) Epling (Muña) }\end{array}$ & 46.15 & 50.01 & 80.77 & 100.092 \\
\hline Muehlenbeckia volcanica & & & & \\
\hline (Bentham) Endlicher & 11.53 & 7.69 & 88.46 & 415.451 \\
\hline (Mullaca) & & & & \\
\hline $\begin{array}{l}\text { Piper elongatum M. Vahl } \\
\text { (Matico) }\end{array}$ & 22.22 & 55.56 & 85.19 & 158.006 \\
\hline
\end{tabular}

De acuerdo a los resultados de la Tabla 5, Acicarpha tribuloides Jussieu (Estrella Kisca) y Gamochaeta spicata (Queto - Queto) se consideran muy tóxicos con una $\mathrm{CL}_{50}$ de 19.639 y 58.104 respectivamente. Por otro lado, la especie Minthostachys glabrescens (Bentham) Epling (Muña)se encuentra justo en el límite para ser considerada muy tóxica con una $\mathrm{CL}_{50}$ de 100.092 y las especies consideradas moderadamente tóxicas son Muehlenbeckia volcanica (Bentham) Endlicher (Mullaca) y Piper elongatum M. Vahl (Matico) con $\mathrm{CL}_{50}$ de 415.451 y 158.006 respectivamente.

\section{Discusión}

Con respecto al análisis fitoquímico cualitativo y espectrofotométrico Uvvis de las hojas de Acicarpha tribuloides Jussieu (Estrella Kisca), no se han reportado investigaciones sobre el contenido de metabolitos secundarios en la especie investigada realizada por otros autores. En nuestro caso, se determinó la presencia de flavonoides, compuestos fenólicos, resinas, alcaloides, taninos y saponinas 
Existen pocos estudios sobre el análisis fitoquímico cualitativo realizado en el extracto etanólico de las hojas de Gamochaeta spicata (Queto-Queto). Los resultados de nuestra investigación coinciden con lo reportado por Lagos y Quinto (2018) que identificaron en la especie vegetal la presencia de flavonoides, compuestos fenólicos, resinas, alcaloides, taninos y saponinas.

En referencia al tamizaje fitoquímico realizado a la especie Minthostachys glabrescens (Bentham) Epling (Muña) no se tuvieron coincidencias con otros estudios de la misma especie; sin embargo, Ambuludi y Cepeda (2015) y Lavado et al. (2021) que realizaron investigaciones en la especie Minthostachys mollis que corresponde al mismo género que la investigada, reportaron en su composición la presencia de saponinas, flavonoides, aceite esencial, alcaloides y taninos, resultados similares a los obtenidos en este estudio.

En el análisis fitoquímico cualitativo y espectrofotométrico Uv-vis en el extracto etanólico de las hojas de Muehlenbeckia volcanica (Bentham) Endlicher (Mullaca), se encontraron flavonoides, compuestos fenólicos, alcaloides, taninos, saponinas y lactonas sesquiterpénicas. No se encontraron estudios en la misma especie, no obstante, Ana Colina (2016) reportó en su estudio de la especie Muehlenbeckia hastulata (J.E.Sm) I.M. Johnst la presencia, en mayor proporción de flavonoides, saponinas y taninos (Colina, A., 2016)

Los hallazgos del estudio fitoquímico preliminar y espectrofotométrico Uv-vis de las hojas de Piper elongatum M. Vahl (Matico) mostraron una abundante cantidad de flavonoides, compuestos fenólicos, resinas, alcaloides, taninos y saponinas. En concordancia con la investigación realizada por Valdez, R. (2014) que reporta en la misma especie la presencia de fenoles, taninos, flavonoides y alcaloides. Asimismo, hay coincidencia con los reportes efectuados por Arroyo, J. et al. (2012) que identificaron en la especie investigada, compuestos fenólicos, alcaloides y flavonoides.

De las cuatro cepas bacterianas evaluadas se observa que Salmonella typhimurium es la más resistente al efecto antibacteriano de las cinco especies vegetales estudiadas, a excepción de Acicarpha tribuloides Jussieu (Estrella Kisca) y la muña con resultados positivos. Un estudio realizado con muestras de Salmonella typhimurium evidencian resistencia a múltiples antibióticos como ampicilina, cloranfenicol, estreptomicina, sulfamidas, siendo el principal mecanismo de resistencia la transferencia de información genética dentro del género (De Toro y col., 2014), lo cual podría explicar también la resistencia a los principios activos de los extractos de especie vegetales.

La ausencia de efecto antibacteriano de Acicarpha tribuloides Jussieu (Estrella Kisca) frente a gram positivos se puede deber a la presencia de peptidoglicano en la pared celular es mayor en comparación a los gram negativos. Resultados diferentes fueron hallados por Jara V (2017), quien encontró actividad antibacteriana frente a Streptococcus mutans (bacteria gram positiva). No se encontraron otros estudios similares en esta especie vegetal. Según el estudio fitoquímico cualitativo, el extracto contiene flavonoides y compuestos fenólicos a los cuales se les atribuye el efecto antimicrobiano. 
Respecto a Gamochaeta spicata (Queto-Queto), en el estudio realizado por Andrade, (2015) que trabaja con aceites esenciales de Gnaphalium pellitum donde se encuentra el efecto antibacteriano frente a Staphylococcus aureus.

No se encontraron estudios con Minthostachys glabrescens (Bentham) Epling (Muña). En el estudio realizado por Torrenegra-Alarcón y col. 2016, evaluaron la actividad antibacteriana de la especie vegetal Minthostachys mollis, cercana a la especie vegetal del presente estudio, hallando efecto antibacteriano frente a Staphylococcus aureus y Escherichia coli ATCC. Lo cual coincide con los resultados del presente estudio. Mostrando adicionalmente actividad frente a Estreptococos pneumoniae y Salmonella typhimurium.

Muehlenbeckia volcanica (Bentham) Endlicher (Mullaca), respecto al efecto antibacteriano frente a Staphylococcus aureus el presente estudio coincide con el estudio realizado por Lagos y Quinto (2018). No se encontraron antecedentes para las otras especies bacterianas.

Piper elongatum M. Vahl (Matico), sobre el efecto antibacteriano frente a Staphylococcus aureus el presente estudio coincide con lo hallado por Zuta N. (2014), que trabajo con la variedad Piper angustifolium quien encontró inhibición con altas concentraciones frente Staphylococcus aureus con resistencia múltiple. Respecto a las demás especies bacterianas no se encontraron antecedentes de estudios.

Según el perfil fitoquímico de las especies vegetales en estudio (tabla 1), todas presentan flavonoides y compuestos fenólicos, los cuales según diversos estudios contienen efecto antibacteriano. Lo cual estaría relacionado con la existencia de grupos hidroxilo $\left(\mathrm{OH}^{-}\right)$y cierto grado de lipofilicidad. (Laks y Pruner, 1989). Así también los fungicidas comerciales, tienen su efecto por la acidez del grupo hidroxilo por desacoplamiento de la fosforilación oxidativa. Los protones son conducidos a través de la membrana mitocondrial destruyendo el diferencial de protones producidos en el transporte electrónico requerido para la formación de ATP. En compuestos fenólicos con un grado de lipofilicidad, mientras mayor sea su número de grupos hidroxilos, resultarán más eficientes desacopladores, ya que, podrían transferir más protones por molécula. Dado que las bacterias no poseen mitocondrias, este mismo mecanismo es el que podría estar operando en la actividad antibacteriana ejercida por los flavonoides, pero a nivel de membrana citoplasmática. (Hammond y Lambert, 1981).

Como se puede observar en la tabla 5, la $\mathrm{CL}_{50}$ de Acicarpha tribuloides Jussieu (Estrella Kisca) es de $19.639 \mathrm{ug} / \mathrm{mL}$ lo que indica que este extracto es muy tóxico debido a que se encuentra por debajo del valor de $100 \mathrm{ug} / \mathrm{mL}$. Para Gamochaeta spicata (Queto - Queto), la $\mathrm{CL}_{50}$ es de $50.104 \mathrm{ug} / \mathrm{mL}$ lo que demuestra que este extracto es muy tóxico debido a que se encuentra por debajo del valor de $100 \mathrm{ug} / \mathrm{mL}$. Luego se tiene que, para Minthostachys glabrescens (Bentham) Epling (Muña), la $\mathrm{CL}_{50}$ es de $100.092 \mathrm{ug} / \mathrm{mL}$ lo que indicaría que este extracto es moderadamente tóxico. Por otro lado, se tiene a Muehlenbeckia volcanica (Bentham) Endlicher (Mullaca) cuya $\mathrm{CL}_{50}$ es de $415.451 \mathrm{ug} /$ $\mathrm{mL}$ que es el valor más alto entre todas las especies estudiadas lo que indicaría que este extracto está considerado como moderadamente tóxico y más aún, es el menos tóxico entre todas las especies estudiadas. Finalmente se tiene el valor de $\mathrm{CL}_{50 \text { de }}$ Piper elongatum $\mathrm{M}$. Vahl (Matico) que es de $158.006 \mathrm{ug} / \mathrm{mL}$ lo que indicaría que este extracto está considerado como moderadamente tóxico. 
Es importante resaltar que las especies vegetales estudiadas, no siempre muestran una concentración letal baja que demostraría su alta bioactividad o citotoxicidad y esto podría explicarse a las condiciones ambientales de su hábitat natural (tipo de tierra, $\mathrm{pH}$, altura, humedad y otros) que influyen en la presencia y concentración de determinados metabolitos secundarios o principios activos. (Aranda-Ventura J. et al., 2018)

Por otro lado, la ausencia de citotoxicidad frente a Artemia salina indicaría que la planta estudiada podría ser bien tolerada por los sistemas biológicos, pero para constatar esto, siempre se puede implementar más estudios como pruebas in vivo. Asimismo, si la prueba con Artemia resulta positiva, es decir se verifica la citotoxicidad de los extractos, esto abre la posibilidad de evaluar si existe o no el efecto farmacológico que se esté buscando implementando ensayos in vivo u otros. (Aranda-Ventura J. et al., 2018)

Plazas E. et al. (2008) en su estudio "Flavonoides aislados de las inflorescencias de Piper hispidicum Kunth (Piperaceae) y derivados acetilados" reporta que los compuestos naturales y sus derivados que fueron sometidos al ensayo con Artemia salina mostraron que el flavonoide 5-hidroxi-7metoxiflavanona presentó una $\mathrm{CL}_{50}$ de $1.8 \mathrm{ug} / \mathrm{mL}$ que es una concentración letal bastante baja que la que muestra este metabolito, en comparación con el extracto estudiado que aun siendo del mismo género, su toxicidad es moderada. Esto puede indicar que los extractos de las piperáceas son en general menos tóxicos que sus metabolitos. En comparación con nuestro estudio, el extracto de la especie Piper elongatum M. Vahl (Matico) mostró una $\mathrm{CL}_{50}$ de $158.006 \mathrm{ug} / \mathrm{mL}$, muy superior al metabolito investigado por Plazas E. et al.

Los estudios con enfoque etnobotánico como el realizado por Desmarchelier et al. (1995) sobre plantas utilizadas en la medicina tradicional de un grupo indígena Takana de la Amazonía peruana, muestra la riqueza de este grupo étnico, utilizando como uno de los bioensayos el de Artemia salina Leach, determinándose entre las 50 plantas descritas, la concentración letal media por el método estadístico Probit de dos especies vegetales, Gamochaeta spicata (Queto - Queto), cuyos extractos metanólico y diclorometánico mostraron una $\mathrm{CL}_{50}$ mayor a $1000 \mathrm{ug} / \mathrm{mL}$ en ambos casos y para la especie Piper angustifolium R.et P., se obtuvo una $\mathrm{CL}_{50}$ de $719 \mathrm{ug} / \mathrm{mL}$ para el extracto metanólico y de $220 \mathrm{ug} / \mathrm{mL}$ para el extracto diclorometánico. De acuerdo al criterio de actividad propuesto por Meyer et al., si se obtiene una $\mathrm{CL}_{50}$ menor a $1000 \mathrm{ug} / \mathrm{mL}$ es indicativo de un extracto activo. Además, Desmarchelier et al., determinó que, Gamochaeta spicata (Queto - Queto) no resultó activo, mientras que Piper angustifolium en ambos tipos de extractos, metanólico y diclorometánico, mostraron ser activos. A diferencia de este estudio, en nuestra investigación, el extracto etanólico de Gamochaeta spicata (Queto Queto), mostró ser activo, con una $\mathrm{CL}_{50}$ de $58.4 \mathrm{ug} / \mathrm{mL}$., al igual que el extracto de Piper elongatum $\mathrm{M}$. Vahl (Matico), del mismo género que la especie estudiada por Desmachelier et al., que también mostró ser muy activo con una $\mathrm{CL}_{50}$ de $158.006 \mathrm{ug} / \mathrm{mL}$.

En cuanto al género Piper específicamente, en Colombia se usa en el tratamiento de diarreas, disentería, dolores de estómago, caries dentales, cicatrizante, para prevenir las caries y como estimulante ya que produce un efecto narcótico y se dice que tiene una acción similar a la coca. También se reporta su uso en las comunidades indígenas del departamento de Chocó como antiinflamatorios, analgésicos, antirreumáticos y contra la picadura de serpientes. (Plazas, 2008).

Los resultados del presente trabajo de investigación permiten conocer aún más sobre las propiedades farmacológicas de especies vegetales que crecen en la zona altoandina y amazónica del Cusco, la mayoría aún poco estudiadas, sobre todo en relación 
a su actividad antibacteriana y la citotoxicidad, por lo que se sugiere profundizar el estudio de especies nativas y ahondar en el conocimiento de sus metabolitos activos, siempre en la búsqueda de sustancias activas con posibilidades de uso en la industria farmacéutica.

Las implicancias de los resultados de esta investigación se encuentran sobre todo en que proporciona los datos que sustentan el uso de estas plantas desde el punto de vista del conocimiento popular transmitido a través del tiempo de una generación a otra en un país donde la fitoterapia es una de las formas más arraigadas en la población, y de esta forma promover su utilización segura y eficaz.

\section{Conclusiones}

Se llegaron a las siguientes conclusiones: el análisis fitoquímico cualitativo y espectrofotométrico UV-Vis, demostró que las plantas investigadas contienen en mayor cantidad compuestos fenólicos, flavonoides, taninos y saponinas. Con respecto a la actividad antibacteriana, el extracto etanólico de las hojas de Queto-Queto y Mullaca tienen efecto frente a Staphylococcus aureus, Escherichia coliy Streptococos pneumoniae, mientras que, el extracto etanólico de las hojas de Matico actúa frente Streptococos pneumoniae y Staphylococcus aureus. En el caso del extracto etanólico de la Estrella Kisca y el aceite esencial de Muña dieron positivo frente a Salmonella typhimurium y Escherichia coli. La Muña también dio positivo a Streptococcus pneumoniae. La prueba de citotoxicidad con Artemia salina, demostró que, los extractos de Acicarpha tribuloides Jussieu (Estrella Kisca) y Gamochaeta spicata (Queto - Queto) son muy tóxicos con una CL50 de 19.639 y 58.104 respectivamente. Por otro lado, los extractos considerados moderadamente tóxicos son los de Minthostachys glabrescens (Bentham) Epling (Muña), Muehlenbeckia volcanica (Bentham) Endlicher (Mullaca), Piper elongatum M. Vahl (Matico) con $\mathrm{CL}_{50}$ de 100.092, 415.451 y 158.006 respectivamente. Todas las especies investigadas en el presente estudio son consideradas prometedoras desde el punto de vista de sus propiedades farmacológicas, en la búsqueda de nuevas sustancias activas para el desarrollo de nuevos medicamentos.

\section{Agradecimientos}

El presente estudio fue financiado por la Universidad Nacional de San Antonio Abad del Cusco a través de fondos canon. Agradecemos a los asistentes de investigación que colaboraron en la ejecución del proyecto.

\section{Conflicto de intereses}

Los autores declaramos no tener ningún conflicto de intereses. 


\section{Referencias}

Agapito, F. y Sung, I. (2005). Fitomedicina: 1100 Plantas Medicinales. Isabel.

Aguirre, L. G., Pereyra-Aguilar, P., Silva-Arrieta-Ontaneda, I., Alarcón-Urbina, M., Palacios-Quintana, M., Medina-Salazar, H., \& Luján-Carpio, E. (2016). Consumo de plantas medicinales en usuarios del "Centro Integral del Adulto Mayor" de la Punta-Callao (Perú). Revista de Fitoterapia, 16(2), 165-175. https://dialnet. unirioja.es/servlet/articulo? codigo $=6088954$

Alfonso, M., Fernández, L., González, N., Avilés, R. (2000). La Achira (Canna edulis Ker.) y su potencialidad en el control de plagas. Ponencia. XII Fórum de Ciencia y Técnica. NIFAT, La Habana. Cuba.

Ambuludi, D., \& Cepeda, N. (2015). Estudio fitoquímico preliminar del follaje de minthostachys mollis cultivada en Quito. [Trabajo de titulación, UTMACH, Unidad Académica de Ciencias Química y de la Salud, Machala, Ecuador]. Repositorio institucional UTMACH. http://repositorio.utmachala.edu.ec/handle/48000/3704

Andrade, W. (2015). Composición de los aceites esenciales de las hojas de conyza bonariensis, gnaphalium pellitum y achyrocline satureioides, por cg-em $y$ evaluación de la actividad antibacteriana y antioxidante. [Tesis de titulación, Pontificia Universidad Javeriana]. Repositorio institucional universidad Javeriana. https://repository.javeriana.edu.co/handle/10554/20619

Aranda-Ventura, J., Villacrés-Vallejo, J., Nuñez-Tuesta, L., Marín-Sisley, P., NonatoRamírez, L. \& González-Aspajo G. (2018). Evaluación de la Bioactividad de plantas medicinales cultivadas en el Perú usando la prueba de letalidad de Artemia salina. Revista Peruana de Medicina Integrativa. 3(3). http://dx.doi.org/10.26722/ rpmi.2018.33.93

Arroyo, J., Hañari, H., Tinco, A., Baca, D., Domínguez, L., Buendía, J. (2012). Efecto antihipertensivo del extracto Piper aduncum "matico" sobre la hipertensión inducida por L-NAME en ratones. Revista Anales de la Facultad de Medicina. Universidad Nacional Mayor de San Marcos. 73(4) oct/dic. http://www.scielo. org.pe/scielo.php?script $=$ sci arttext\&pid $=$ S1025-55832012000400002

Brack Egg, A. (1999). Diccionario enciclopédico de plantas útiles del Perú. Centro Bartolomé de las Casas.

Bussmann, R. W., \& Sharon, D. (2016). Uso de Plantas medicinales en los Andes Norte del Perú. Ethnobotany Research and Applications, 15(1), 1-293. https:// ethnobotanyjournal.org/index.php/era/article/view/1285 
Canal Gallego, D. (2011), Cestrum de Colombia (Solanaceae): estudio taxonómico de las especies de tricomas simples. [Tesis de titulación, Universidad Nacional de Colombia]. Repositorio institucional UNAL. https://repositorio.unal.edu.co/ handle/unal/7722

CIPI - Centro de Investigación de la Producción Industrial (1994). Catálogo de Plantas Medicinales. Universidad de Lima. Facultad de Ingeniería Industrial. Lima.

Claus, E. (1980). Farmacognosia. El Ateneo. Buenos Aires, Argentina.

Colina, A. (2016). Análisis fitoquímico, determinación cualitativa y cuantitativa de flavonoides y taninos, actividad antioxidante, antimicrobiana de las hojas de "Muehlenbeckia hastulata (J.E.Sm) I.M. Johnst" de la zona de Yucay (Cusco). [Tesis de titulación. Universidad Nacional Mayor de San Marcos. Perú]. Repositorio institucional cybertesis. https://cybertesis.unmsm.edu.pe/ handle/20.500.12672/7121

CYTED Programa Iberoamericano de Ciencia y Tecnología para el Desarrollo (1995). Manual de Técnicas de Investigación. Búsqueda de Principios Activos en Plantas de la Región. CYTED.

De Toro, M., Seral, C., Rojo-Bezares, B., Torres, C., Castillo, F. J., y Sáenz, Y. (2024). Resistencia a antibióticos y factores de virulencia en aislados clínicos de Salmonella enterica. Enfermedades Infecciosa y Microbiología Clínica, 32(1), 4-10. https:// doi.org/10.1016/j.eimc.2013.03.006

Desmarchelier, C., Mongelli, E., Coussio, J., Giulietti, A., Ciccia, G. (1995). Etnobotánica y Bioactividad de Plantas medicinales utilizadas por un Grupo Indígena Takana de la Amazonía Peruana. Acta Farmacia Bonarense 14(3), 195-208. http://www. latamjpharm.org/trabajos/14/3/LAJOP 14 3 _ 116 6996D8765SA.pdf

Font Quer, P. (1982). Diccionario de Botánica (2ª ed.). Península.

Granados, R. y Villaverde, C. (1997). Microbiología. Paraninfo.

Huamantupa, I., Cuba, M., Urrunaga, R., Paz, E., Ananya, N., Callalli, M., Pallqui, N., \& Coasaca, H. (2011). Riqueza, uso y origen de plantas medicinales expendidas en los mercados de la ciudad del Cusco. Revista Peruana de Biología, 18(3), 283-292. http://www.scielo.org.pe/scielo.php?pid=S1727$99332011000300004 \&$ script $=$ sci_arttext

Jara V. (2017). Efecto antimicrobiano in vitro de acicarpha tribuloides juss (estrella kiska), frente A Streptococcus mutans. [Tesis de titulación, Universidad Andina del Cusco]. Repositorio institucional UAC. https://repositorio.uandina.edu.pe/ handle/20.500.12557/1806 
Lagos, D. \& Quinto, R. (2018). Efecto antibacteriano del extracto etanólico de las hojas de Muehlenbeckia volcánica (benth.) endl. (mullaca) en cepas de Staphylococcus aureus ATCC 6538, in vitro. [Tesis de titulación, UIGV. Lima Perú]. Repositorio institucional Universidad Inca Garcilaso de la Vega. http://repositorio.uigv.edu. pe/handle/20.500.11818/3074

Laks, P. \& Pruner, M. (1989). Flavonoid biocides: Structure/activity relations of flavonoid phytoalexin analogues. Phytochemistry, 28; 87.

Lavado, I., Andamayo, D., Castillo, D., Junchaya, V. (2021). Evaluación preliminar de 10 plantas medicinales del Valle del Mantaro mediante el método cualitativo (fitoquímico) para uso farmacéutico. Revista Visionarios en Ciencia y Tecnología 6:38-48. https://revistas.uroosevelt.edu.pe/index.php/VISCT/article/view/88/135

Organización Mundial de la Salud (OMS) (2002). Estrategia de la OMS sobre medicina tradicional 2002-2005. Programa de medicina tradicional. OMS. http://apps. who.int/iris/bitstream/handle/10665/67314/WHO_EDM_TRM_2002.1_spa. pdf;jsessionid=4F845D3E2D110A0A71F8C6650857F7D9?sequence $=1$

Organización Mundial de la Salud (OMS) (2000) Pautas generales para las metodologías de investigación y evaluación de la medicina tradicional. OMS. https://apps.who. int/iris/handle/10665/67719

Palacios, J. (1993). Plantas medicinales nativas del Perú. (1 ${ }^{a}$ Ed). CONCYTEC. ABBSA. pp. 50-53.

Plazas, E., Cuca, L., Delgado, A., Wilman, A. (2008). Flavonoides aislados de las Inflorescencias de Piper hispidum Kunth (Piperaceae) y Derivados Acetilados. Revista Colombiana de Química, 37(2), 135-144 http://www.scielo.org.co/scielo. php?pid $=$ S0120-28042008000200002\&script $=$ sci abstract\&tlng $=$ en

Rojas, J. (1986). Farmacobotánica. Guía de Descripciones Morfológicas y Ubicación Sistemática de las Especies Vegetales del Jardín Botánico de la facultad de Farmacia y bioquímica. Universidad Nacional Mayor de San Marcos. Lima Perú.

Salaverry, O. (2005). La complejidad de lo simple: Plantas medicinales y sociedad moderna. Revista Peruana de Medicina Experimental y Salud Publica, 22(4), 245-246. http://www.scielo.org.pe/scielo.php?script=sci arttext\&pid=S1726-46342005000400001

Schulz V., Hansel R., y Tyler V., (2002), Fitoterapia Racional. Guía de Fitoterapia para las Ciencias de Salud. (4 ${ }^{\text {a }}$ ed.). Manole Ltda., Tambore, Brasil, pp. 98-101.

Torrenegra-Alarcón, M., Granados-Conde, C., Durán-Lengua, M., León-Méndez, G., Yáñez-Rueda, X., Martínez, C., \& Pájaro-Castro, N. (2016). Composición Química y Actividad Antibacteriana del Aceite Esencial de Minthostachys mollis. 
Orinoquia, 20(1), 69-74. https://www.redalyc.org/pdf/896/89647074008.pdf

Trease-Evans (1991). Farmacognosia. (13ª. Ed.). Interamericana. México.

U.S.P. (2015). United States Pharmacopeia. Volumen 38. Formulario NF 33.

Valdez, R. (2014). Contenido de fenoles totales y flavonoides totales en Oenothera rosea Ait "yawar suqu", Baccharís salicifolia R\&P "chilca" y Piper elongatum Vahl "matico". [Tesis de titulación, Universidad Nacional de San Cristóbal de Huamanga]. Repositorio institucional UNSCH. http://repositorio.unsch.edu.pe/ handle/UNSCH/2502

Woods, G. \& Washington, J. (1995). Antibacterial susceptibility test: dilution and diskdiffusion methods. In Manual of clinical microbiology. ( $6^{\mathrm{a}}$ ed.). American society of Microbiology.

Zuta, N. (2014). Actividad antibacteriana in vitro de extractos de piper angustifolium (matico) y Matricaria chamomilla (manzanilla) en cepas de Staphylococcus aureus con resistencia múltiple. [Tesis de titulación, Universidad Nacional del Callao. Perú]. Repositorio institucional UNAC. http://repositorio.unac.edu.pe/ handle/20.500.12952/943 